\title{
On the Largest Prime Divisor of an Odd Perfect Number. II
}

\author{
By Peter Hagis, Jr. and Wayne L. McDaniel
}

\begin{abstract}
It is proved here that every odd perfect number has a prime factor greater than 100110 .
\end{abstract}

If $n$ is an element of the (possibly empty) set of odd perfect numbers, then it is well known that

$$
n=p_{0}^{\alpha} 0 \cdot p_{1}^{\alpha} \cdots p_{t}^{\alpha}
$$

where the $p_{i}$ are distinct primes, $p_{0} \equiv \alpha_{0} \equiv 1(\bmod 4)$, and $2 \mid \alpha_{i}$ if $i>0$. In [2], it was proved that at least one of the $p_{i}$ exceeds 11200 . Our purpose here is to improve this bound by proving the following:

THEOREM. If $n$ is odd and perfect, then $n$ has a prime factor which exceeds 100110.

The method of proof is similar to that employed in [2], and we shall not give the details here. We shall, however, explain our strategy and exemplify the arguments which are used. The complete proof [1] has been deposited in the UMT file.

The proof is by reductio ad absurdum. Thus, we assume that $p_{i}<100110$ for every $p_{i}$ in (1) and show that this assumption is untenable. Since $n$ is perfect $\sigma(n)=$ $2 n$, and since $\sigma(n)$ is multiplicative,

$$
2 n=\prod_{i=0}^{t} \sigma\left(p_{i}^{\alpha}\right)=\prod_{i=0}^{t} \prod_{d} F_{d}\left(p_{i}\right) .
$$

Here $F_{d}$ is the $d$ th cyclotomic polynomial, and $d$ runs over the divisors of $\alpha_{i}+1$ which exceed 1. $d$ assumes the value 2 if and only if $i=0$. We see immediately that the set of $p_{i}$ in (1) is identical with the set of odd prime divisors of the $F_{d}\left(p_{i}\right)$ in (2). In particular, recalling our assumption, we note that all the prime factors of each $F_{d}\left(p_{i}\right)$ must be less than 100110 .

For a given odd prime $p$ we shall say that the prime $Q$ is $(p ; 100110)$-acceptable or simply p-acceptable if every prime divisor of $F_{Q}(p)$ is less than 100110. According to a result of Kanold [3, (21) Satz], if $Q>50053$, then $Q$ is unacceptable for every odd prime. We shall say that $p$ is inadmissible if no $Q$ is $p$-acceptable. ( $Q=2$ is taken into consideration only if it is possible that $p=p_{0}$.)

Our proof is in two stages, and we show first that $n$ is not divisible by certain "small" primes.

LEMмA. If every prime in the factorization of the odd perfect number $n$ is less

Received April 25, 1974; revised September 24, 1974.

AMS (MOS) subject classifications (1970). Primary $10 \mathrm{~A} 20$.

Copyright $\odot 1975$, American Mathematical Society 
than 100110, then $n$ is not divisible by any prime in the set $V$ where $V=\{3,5,7,11$, $13,19,23,31,37,61,127,131,151,1093$ \}.

The proof of this lemma goes as follows: Assuming that $p \mid n$ (which we wish to disprove), we find all $p$-acceptable primes and then factor $F_{Q}(p)$; from (2) $F_{Q}(p) \mid 2 n$ for at least one $p$-acceptable prime $Q$ and each odd prime divisor of $F_{Q}(p)$ divides $n$; for each acceptable $F_{Q}(p)$, a single prime divisor is selected and its acceptable primes are determined; this procedure is iterated and a finite tree is generated (finite, since each prime on which we branch is less than 100110 and its acceptable primes are bounded by 50053); each path through the tree terminates at a node corresponding to either an inadmissible prime or some other contradiction so that $p \nmid n$. A priori, a third type of terminal node might be encountered-one corresponding to an admissible prime $r$ such that every odd prime divisor of each $r$-acceptable cyclotomic number has already been branched upon on the path joining $p$ to $r$, in which case our procedure fails. We encountered no such nodes, and fortunately most of the trees generated were small. We illustrate by showing that neither 1093 nor 151 divides $n$, and begin by proving:

(A) If $613 \mid n$, then $613=p_{0}$. The only odd 613 -acceptable primes are 3 and 5 and $F_{3}(613)=3 \cdot 7 \cdot 17923, F_{5}(613)=131 \cdot 20161 \cdot 53551$. Therefore, if $613 \mid n$ and $613 \neq p_{0}$ then $17923 \mid n$ or $53551 \mid n$. Since both 17923 and 53551 are inadmissible, our result follows.

(B) $1093 \nmid n$. Only 2 is 1093 -acceptable and $F_{2}(1093)=2 \cdot 547$. Therefore, if $1093 \mid n$, then $547 \mid n$ also. Only 3 is 547 -acceptable and $F_{3}(547)=3 \cdot 163 \cdot 613$. Therefore, $1093=p_{0}$ and (from (A)) $613=p_{0}$. We have reached a contradiction.

(C) $151 \nmid n$. For, only 3 is 151 -acceptable and $F_{3}(151)=3 \cdot 7 \cdot 1093$, so that if $151 \mid n$, then $1093 \mid n$, which contradicts (B).

To describe the second stage of our proof, we need several more definitions. Let $q$ be the smallest prime divisor of $n$ and let $W(q)$ denote the set of primes which are not less than $q$. For a given prime $p$, we shall say that the prime $Q$ is $(p ; q ; 100110)$ feasible or simply $(p, q)$-feasible if $Q$ is $p$-acceptable and if every odd prime divisor of $F_{Q}(p)$ belongs to the set $W(q) \cap V^{\prime}$ where $V^{\prime}$ denotes the complement of $V$ with respect to the set of all primes. (Of course, for each $p_{i}$ in (2), each prime divisor of $\alpha_{i}+1$ must be $\left(p_{i}, q\right)$-feasible.) If $p$ cannot be $p_{0}$, we omit $Q=2$ from consideration. If no $Q$ is $(p, q)$-feasible, we shall say that $p$ is $q$-impossible.

Now, according to the table in [4], $q<307$ since otherwise $n$ would have a prime factor which exceeds 100549. But (see [1]) except for the elements of the set $T=\{17,41,59,67,71,79,89,101,149,167,173,197,293\}$ every odd prime $r$ less than 307 is either $r$-impossible or belongs to $V$, so that $q \in T$. Using basically the method described above for the proof of our lemma, we complete the proof of our theorem by showing that no prime in $T$ is $q$. We illustrate by proving:

(a) $q \neq 17$. For, only 3 and 5 are $(17,17)$-feasible. But $F_{3}(17)=307$, only 5 is $(307,17)$-feasible, $1051 \mid F_{5}(307)$, and 1051 is 17 -impossible. $F_{5}(17)=88741$, only 2 is $(88741,17)$-feasible, $44371 \mid F_{2}(88714)$, and 44371 is 17 -impossible.

Concluding Remarks. If $P$ is the largest prime divisor of the odd perfect number $n$, then a "good" bound on $P$ is very helpful if one is investigating such questions as 
"How large is $n$ ?" or "How many prime divisors does $n$ have?". This is the motivation for the present paper. It is obvious that by modifying appropriately the definitions of $p$-acceptable, $(p, q)$-feasible, etc., and expending the requisite effort and computer time, one could very probably improve our lower bound on $P$. The present investigation consumed approximately 6.5 hours of CDC 6400 time, most of which was devoted to verifying that, for each prime on which we branched, almost all $Q \leqslant 50053$ were unacceptable. The complete factorizations of all $p$-acceptable $F_{Q}(p)$ encountered are given in Table $I$ in [1]. We do not intend to pursue this research further and would hope that if someone else does that he aim for a lower bound on $P$ of at least $10^{6}$.

Acknowledgment. The authors would like to express their appreciation to the referee for his detailed and helpful report concerning this research. It is at his suggestion that the complete proof [1] has been deposited in the UMT file and that the present note is being published here.

Department of Mathematics

Temple University

Philadelphia, Pennsylvania 19122

Department of Mathematics

University of Missouri-St. Louis

St. Louis, Missouri 63121

1. P. HAGIS, JR. \& W. L. MCDANIEL, "A proof that every odd perfect number has a prime factor greater than 100110." (Copy deposited in UMT file.)

2. P. HAGIS, JR. \& W. L. MCDANIEL, "On the largest prime divisor of an odd perfect number," Math. Comp., v. 27, 1973, pp. 955-957. MR 48 \#855.

3. H. J. KANOLD, “Untersuchungen über ungerade vollkommene Zahlen," J. Reine Angew. Math., v. 183, 1941, pp. 98-109. MR 3, 268.

4. K. NORTON, "Remarks on the number of factors of an odd perfect number," Acta. Arith., v. 6, 1960, pp. 365-374. MR 26 \#4950. 